\title{
Redução da mobilidade funcional e da capacidade cognitiva no diabetes melito tipo 2
}

\author{
Reduction of functional mobility and cognitive \\ capacity in type 2 diabetes mellitus
}

Mari Cassol Ferreira', Joana Tozatti², Silvia Maria Fachin'2, Patricia Pereira de Oliveira ${ }^{3}$, Rosa Ferreira dos Santos ${ }^{4}$, Maria Elisabeth Rossi da Silva ${ }^{4}$

${ }^{1}$ Programa de Endocrinologia e Metabologia, Faculdade de Medicina da Universidade de São Paulo (FMUSP). Faculdade de Medicina da Universidade Comunitária Regional de Chapecó (Unochapecó), Chapecó, SC, Brasil 2 Universidade Comunitária Regional de Chapecó (Unochapecó)، Chapecó, SC, Brasil

${ }^{3}$ Instituto Fernandes Figueira,

Fundação Oswaldo Cruz (IFF/ Fiocruz). Faculdade de Medicina da Unochapecó, Chapecó, SC, Brasil ${ }^{4}$ Laboratório de Carboidratos, LIM18. Disciplina de Endocrinologia e Metabologia, Hospital das Clínicas da Faculdade de Medicina da Universidade de São Paulo (HCFMUSP), São Paulo, SP, Brasil

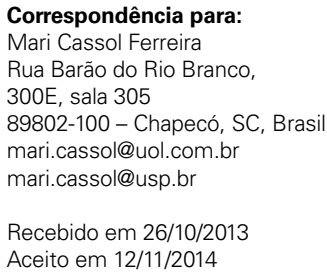

DOI: 10.1590/0004-2730000003097

\section{RESUMO}

Objetivos: Avaliar a mobilidade funcional e sua relação com a capacidade cognitiva em pacientes com diabetes tipo 2 (DM2) entre 50 e 65 anos de idade, e com menos de 10 anos de diagnóstico. Materiais e métodos: Estudo observacional, analítico e transversal envolvendo indivíduos não diabéticos e pacientes com DM2 com controle glicêmico inadequado, selecionados por amostra de conveniência. Em ambos os grupos, foram aplicados questionário estruturado, avaliação cognitiva com Miniexame do Estado Mental (MEEM) e teste do relógio (TDR), além da avaliação de mobilidade funcional pelo testeTimed Up \& GO (TUG). Resultados: NoTUG os pacientes com DM2 apresentaram tempo médio de 11,27 segundos versus 9,52 segundos nos controles $(p=0,013)$. A associação entre declínio cognitivo e dismobilidade foi positiva nos indivíduos com DM2 ( $p=0,037)$. No subgrupo que apresentou dismobilidade e declínio cognitivo associados, $18 \%$ eram portadores de DM2 e 1,6\% era do grupo sem DM2 $(p<0,01)$. Conclusões: Pacientes com DM2 apresentaram pior mobilidade funcional e desempenho cognitivo, favorecendo a hipótese de que o DM2 influencia a mobilidade funcional e capacidade cognitiva antes do aparecimento de complicações vasculares ou neuropáticas. Esses dados sugerem que a hiperglicemia é um fator agravante no desempenho de atividades que exijam funções mentais como atenção, orientação e memória de trabalho. Arq Bras Endocrinol Metab. 2014;58(9):946-52

\section{Descritores}

Diabetes tipo 2; cognição; acidentes por quedas; aptidão física

\section{ABSTRACT}

Objectives: The aim of the present study was to evaluate the functional mobility and its relationship to cognitive ability in patients with type 2 diabetes (T2DM), age between 50 and 65 years and under 10 years of diagnosis. Materials and methods: An observational, analytical and cross-sectional study, involving no diabetic and type 2 diabetic individuals with inadequate glycemic control, selected by convenience sampling. In both groups, were administered structured questionnaire and cognitive assessment with Mini-Mental State Examination (MMSE) and the clock drawing test (CDT), besides assessment of functional mobility by the Timed Up \& Go (TUG). Results: InTUG, DM2 patients presented a mean time of 11.27 seconds versus 9.52 seconds $(p=0.013)$. The association between cognitive decline and decrease of mobility was positive in individuals with T2DM ( $p=0.037)$. In the subgroup that showed decrease of mobility and associated cognitive decline, $18 \%$ were patients with DM2 and $1.6 \%$ were individuals without T2DM ( $p$ < 0.01). Conclusions: Patients with T2DM presented worse functional mobility and cognitive performance, supporting the hypothesis that DM2 influence functional mobility and cognitive ability, regardless of neuropathic or vascular complications. These data suggest that hyperglycemia is an aggravating factor in the performance of activities requiring mental functions such as attention, working memory and orientation. Arq Bras Endocrinol Metab. 2014;58(9):946-52

\section{Keywords}

Type 2 diabetes; cognitive decline; accidents from falls; physical fitness 


\section{INTRODUÇÃO}

$\mathrm{O}$ diabetes melito tipo 2 (DM2) exerce grande impacto na saúde pública em decorrência de suas complicações, comprometendo a qualidade de vida das populações por ele acometidas. Uma importante consequência do envelhecimento das pessoas com DM2 é a incapacidades física, especialmente a perda da mobilidade. A habilidade funcional se traduz como um conjunto de elementos como equilíbrio, marcha e coordenação. Assim, a dismobilidade torna esses pacientes mais dependentes, conduz à perda de massa muscular e reduz a expectativa de vida (1). Os fatores predisponentes frequentes em portadores de diabetes incluem: neuropatia periférica, comprometimento visual, redução da função renal, alterações autonômicas e uso de medicamentos (24). Outras características presentes no paciente com DM2 são o sedentarismo e o ganho de peso ( 1 ) que, aliados à dismobilidade, têm como consequência o maior risco de quedas, que aparecem como importantes causas de ferimento não fatal entre pessoas com mais de 50 anos (5). Portanto, a mobilidade funcional é um elemento central da qualidade da saúde, pois prediz o declínio funcional, o uso de serviços de saúde e a morbimortalidade (6).

Evidências apontam para a relação positiva entre DM2 e declínio cognitivo, o qual também está implicado na inabilidade motora, devido ao comprometimento da atenção, da memória e de respostas protetoras, apraxia, desorientação espacial e deterioração das funções executivas e motoras (marcha alterada, desequilíbrio, instabilidade postural, alteração de tônus muscular); além do fato de os pacientes com essas condições não poderem julgar adequadamente sua própria capacidade, tomando atitudes arriscadas que levam a acidentes (7). Os déficits cognitivos, quando somados à presença de hiperglicemia, também podem aumentar a incapacidade física, trazendo dificuldades na realização de atividades da vida diária (AVD). Entretanto, pouco se sabe sobre a relação entre distúrbio cognitivo e incapacidade física nos pacientes com DM2 (8). Estudos prévios sugerem que a alteração cognitivo-motora pode não apresentar uma relação linear com a glicemia, mas sim que pode haver um limite por volta de $270 \mathrm{mg} / \mathrm{dL}$ a partir do qual a função cognitivo-motora começaria a ser afetada (7). Embora se saiba a respeito da hipoglicemia e sua neuroglicopenia, um dos principais obstáculos para a investigação dos efeitos da hiperglicemia sobre o desempenho cognitivo-motor é a ausência de um mecanismo fisiológico claro que explique sua influência negativa sobre o funcionamento cerebral. A identificação de testes mais acurados para o estabelecimento de algoritmos práticos de mensuração da mobilidade funcional tem sido alvo de estudos recentes (9). Entre eles, o histórico sobre quedas (10), o teste Timed Up \& Go (TUG) (11) e a avaliação da capacidade cognitiva com o Miniexame do Estado Mental (MEEM) se mostraram estratégias eficazes. Portanto, nosso estudo propõe avaliar pacientes com DM2 sem controle glicêmico adequado e comparar com controles não diabéticos em relação ao desempenho cognitivo e motor.

\section{MATERIAIS E MÉTODOS}

Trata-se de estudo observacional, analítico e transversal. A população em análise foi de 145 pessoas usuárias do serviço público municipal de saúde, sendo a amostra estudada composta por 118 pessoas, divididas em dois grupos distintos: grupo DM2, formado por 50 pacientes com DM2 e controle glicêmico inadequado, e grupo controle, com 68 indivíduos sem DM2.

A população com DM2 foi composta por homens e mulheres de 50 a 65 anos, com diagnóstico de DM2 confirmado pela pesquisa de resultados laboratoriais prévios nos prontuários e com tempo de diagnóstico inferior a 10 anos. Foram incluídos somente pacientes com glicemia de jejum maior de $200 \mathrm{mg} / \mathrm{dL}$ no dia da entrevista e realização dos testes cognitivos e da mobilidade funcional e com pelo menos uma glicemia de jejum maior de $150 \mathrm{mg} / \mathrm{dL}$ nos 6 meses que antecederam o estudo. Não incluímos hemoglobina glicada (HbAlC) como critério de inclusão, pois necessitávamos priorizar um exame que avaliasse o estado de hiperglicemia no momento da realização dos testes cognitivo-motores. E sabe-se que uma $\mathrm{HbAlC}$ elevada não exclui a possibilidade de grande variabilidade glicêmica. O grupo controle contou com homens e mulheres de mesma faixa etária, sem diabetes de qualquer etiologia, confirmado pela pesquisa de resultados laboratoriais prévios e com glicemia de jejum menor de $99 \mathrm{mg} / \mathrm{dL}$ no dia da entrevista. Os critérios de exclusão foram pacientes analfabetos, tabagistas, etilistas, usuários de drogas psicoativas, com distúrbios visuais ou auditivos incapacitantes, portadores de neuropatias, ou sequelas neurológicas de AVC, bem como pacientes que não concordaram com o termo de consentimento.

O tamanho da amostra foi calculado com base na prevalência de diabéticos no Brasil (12) e na prevalência de quedas de uma população idosa (38\%) (13), com nível de confiança de $95 \%$ e erro tolerável de $5 \%$, 
para uma população de 22.049 indivíduos (homens e mulheres entre 50 e 65 anos residentes no município). A partir desse resultado, foram submetidos à pesquisa 145 pacientes. Após contato inicial com os pacientes (selecionados na sala de espera de um centro de diversas especialidades médicas), determinou-se a glicemia capilar de jejum por meio de glicosímetros. Os valores anteriores da glicemia (até 6 meses) para caracterização da hiperglicemia persistente (controle inadequado da doença) foram obtidos no prontuário do paciente. Foram excluídos 14 indivíduos com DM2 e 13 do grupo controle cujos dados laboratoriais estavam incompletos.

Foi aplicado um questionário padrão (características gerais) e realizadas medidas de peso e altura para cálculo do índice de massa corporal (IMC), que obedeceu à fórmula peso/altura (14). Para a avaliação da mobilidade funcional e da capacidade cognitiva, foram realizados os testes relacionados a seguir.

\section{Metodologia de avaliação funcional}

1) Verificação de ocorrência de quedas no último ano.

2) Foi requisitado ao paciente que levantasse de uma cadeira (de altura usual), sem apoio dos braços. Essa análise foi expressa em dificuldade ou não em realizar a tarefa (15).

3) Teste Timed Up \& Go (TUG), método rápido, fácil e seguro para avaliação da mobilidade funcional (16). A verificação é realizada com uma cadeira, um cronômetro, fita métrica e uma ficha para anotações dos dados. Mede-se o tempo gasto para levantar de uma cadeira (com $45 \mathrm{~cm}$ de altura), andar uma distância de três metros, dar a volta, caminhar em direção à cadeira e sentar-se novamente. O paciente realiza primeiro o teste uma vez para se familiarizar e nenhuma ajuda é dada durante a sua realização (17). Considera-se que quanto maior o tempo gasto para realizar a atividade, mais limitada é a mobilidade (18). De modo geral, pacientes independentes e sem nenhuma alteração de equilíbrio demoram menos de 10 segundos. Por outro lado, pacientes dependentes demoram mais de 20 segundos. Pacientes que demoram de 10 a 20 segundos possuem certo grau de limitação, porém ainda são considerados independentes $(19,20)$.

\section{Metodologia de avaliação da função cognitiva}

1) Miniexame do Estado Mental (MEEM): é um dos testes mais utilizados na prática clínica. Abrange seis áreas da cognição: linguagem, orientação, habilidade construtiva, atenção/cálculo, retenção e evocação. O escore máximo é de 30 pontos. São considerados como declínio cognitivo valores inferiores a 27 para pessoas com mais de 11 anos de escolaridade, inferiores a 22 para pessoas com menos de 11 anos de escolaridade e inferiores a 15 para analfabetos. Considerando que excluímos pacientes analfabetos, a pontuação corte para declínio cognitivo foi de 22 pontos (21).

2) Teste do Desenho do Relógio (TDR): reconhecido como teste de grande utilidade no diagnóstico e seguimento de demências. Pede-se ao paciente que desenhe um relógio marcando 11 horas e 10 minutos, sem tempo cronometrado. Todos os números devem estar representados (14,21). As notas variam de 0 (incapacidade absoluta de desenhar) a 5 (relógio perfeito).

A análise estatística foi realizada com auxílio do programa Statistical Package for the Social Sciences versão 17. Foram descritas as características quantitativas segundo presença ou não de DM2 com o uso de medidas resumo (média, desvio-padrão, mediana, mínimo e máximo) e comparadas pelo teste $t$ de Student e, para as escalas MEEM e teste TUG, foram utilizados os testes Mann-Whitney. As características qualitativas foram descritas pelas de frequências absolutas e relativas e a existência de associação pelos testes qui-quadrado ou testes da razão de verossimilhanças. Foi criada uma regressão linear múltipla para explicar o resultado do teste TUG de acordo com as características dos pacientes, da escala MEEM e da presença ou não de DM2.

Este estudo obedeceu aos critérios de ética preconizados pela resolução n ${ }^{\circ}$ 196/96 do Conselho Nacional de Saúde (CNS) do Ministério da Saúde e foi submetido à aprovação do Comitê de Ética em Pesquisa da Universidade Comunitária da Região de Chapecó (Unochapecó) antes de sua execução. Todos os participantes assinaram o termo de consentimento livre e esclarecido.

\section{RESULTADOS}

As características da população do estudo, de 118 indivíduos, sendo 50 com DM2 e 68 controles, são apresentadas nas tabelas 1 e 2 .

A taxa de quedas no último ano foi de $42 \%$ entre pacientes com DM2 e 33,8\% controles ( $\mathrm{p}=0,364)$. Entretanto, o número médio de quedas entre os grupos foi de 1,57 $\pm 1,07$ nos DM2 e de 2,09 $\pm 1,97$ nos controles $(\mathrm{p}=0,058)$. A dificuldade em levantar da cadeira sem apoio foi presente em $22 \%$ dos pacientes com DM2 e $4,4 \%$ dos controles $(\mathrm{p}=0,004)$. 
Tabela 1. Características sociodemográficas da população em estudo $(n=118)$

\begin{tabular}{|c|c|c|c|c|c|c|c|}
\hline & \multicolumn{2}{|c|}{ DM2 } & \multicolumn{2}{|c|}{ Controles } & \multicolumn{2}{|c|}{ Total } & \multirow{2}{*}{ p } \\
\hline & $\mathbf{n}$ & $\%$ & $\mathbf{n}$ & $\%$ & $\mathbf{n}$ & $\%$ & \\
\hline Sexo & & & & & & & 0,001 \\
\hline Feminino & 56 & 82,4 & 27 & 54,0 & 83 & 70,3 & \\
\hline Masculino & 12 & 17,6 & 23 & 46,0 & 35 & 29,7 & \\
\hline Cor da pele & & & & & & & 0,120 \\
\hline Não branca & 11 & 16,2 & 14 & 28,0 & 25 & 21,2 & \\
\hline Branca & 57 & 83,8 & 36 & 72,0 & 93 & 78,8 & \\
\hline Estado civil & & & & & & & $0,076^{\sharp}$ \\
\hline Casado & 49 & 72,1 & 44 & 89,8 & 93 & 79,5 & \\
\hline Solteiro & 2 & 2,9 & 0 & 0,0 & 2 & 1,7 & \\
\hline Viúvo & 10 & 14,7 & 3 & 6,1 & 13 & 11,1 & \\
\hline Separado & 7 & 10,3 & 2 & 4,1 & 9 & 7,7 & \\
\hline Escolaridade & & & & & & & $0,746^{\sharp}$ \\
\hline EF incompleto & 50 & 73,5 & 38 & 76,0 & 88 & 74,6 & \\
\hline EF completo & 9 & 13,2 & 7 & 14,0 & 16 & 13,6 & \\
\hline EM incompleto & 3 & 4,4 & 3 & 6,0 & 6 & 5,1 & \\
\hline EM completo & 6 & 8,8 & 2 & 4,0 & 8 & 6,8 & \\
\hline Queda no último ano & & & & & & & 0,364 \\
\hline Não & 45 & 66,2 & 29 & 58,0 & 74 & 62,7 & \\
\hline Sim & 23 & 33,8 & 21 & 42,0 & 44 & 37,3 & \\
\hline Total & 68 & 100 & 50 & 100 & 118 & 100 & \\
\hline
\end{tabular}

Resultado do teste qui-quadrado \# Resultado do teste da razão de verossimilhanças. DM2: diabetes melito tipo 2; EM: ensino médio; EF: ensino fundamental.

Tabela 2. Características antropométricas e laboratoriais da população em estudo $(n=118)$

\begin{tabular}{|c|c|c|c|c|c|c|c|c|}
\hline & Diabetes & Média & DP & Mediana & Mínimo & Máximo & $\mathbf{N}$ & $\mathbf{p}$ \\
\hline \multirow[t]{2}{*}{ Idade (anos) } & Controle & 56,21 & 4,31 & 55,5 & 50 & 65 & 68 & 0,056 \\
\hline & DM2 & 57,86 & 4,97 & 58 & 50 & 65 & 50 & \\
\hline \multirow[t]{2}{*}{ Peso (kg) } & Controle & 71,71 & 13,28 & 71 & 45 & 101 & 62 & 0,013 \\
\hline & DM2 & 79,67 & 19,58 & 77,5 & 42 & 169 & 48 & \\
\hline \multirow[t]{2}{*}{ Altura (m) } & Controle & 161,11 & 7,50 & 160 & 147 & 179 & 62 & 0,174 \\
\hline & DM2 & 163,33 & 9,50 & 165 & 132 & 181 & 48 & \\
\hline \multirow[t]{2}{*}{ IMC } & Controle & 27,06 & 4,55 & 27 & 18 & 38 & 62 & 0,034 \\
\hline & DM2 & 29,48 & 7,20 & 28,5 & 19 & 59 & 48 & \\
\hline \multirow[t]{2}{*}{ Glicemia capilar } & Controle & 81,89 & 10,17 & 82 & 56 & 100 & 37 & $<0,001$ \\
\hline & DM2 & 287,92 & 86,45 & 262,5 & 200 & 553 & 50 & \\
\hline \multirow[t]{2}{*}{ Triglicerídeos (mg/dL) } & Controle & 138,58 & 72,75 & 142 & 36 & 364 & 33 & 0,002 \\
\hline & DM2 & 289,08 & 211,64 & 214,5 & 40 & 866 & 26 & \\
\hline \multirow[t]{2}{*}{ HDL (mg/dL) } & Controle & 52,03 & 10,06 & 50 & 33 & 73 & 31 & 0,006 \\
\hline & DM2 & 43,82 & 10,17 & 44,5 & 23 & 61 & 22 & \\
\hline \multirow[t]{2}{*}{ Colesterol T (mg/dL) } & Controle & 211,13 & 31,26 & 209,5 & 139 & 278 & 32 & 0,242 \\
\hline & DM2 & 228,73 & 69,84 & 227,5 & 124 & 463 & 26 & \\
\hline
\end{tabular}

Resultados do teste $t$ de Student. DM2: diabetes melito tipo 2; IMC: índice de massa corpórea; colesterol T: colesterol total. 
O teste TUG foi realizado em $11,27 \pm 3,2$ segundos nos pacientes DM2, tempo superior aos controles, que foi de 9,52 $\pm 3,6$ segundos ( $\mathrm{p}=0,001)$, (Tabela 3 ). Cerca de $30 \%$ e $52,9 \%$ dos pacientes DM2 e controles, respectivamente, levaram menos de 10 segundos para execução do teste $(\mathrm{p}=0,013)$, evidenciando que a maior parte do grupo controle não tinha limitação funcional. Observamos que 22\% dos pacientes DM2 e $57 \%$ dos controles não apresentaram limitação funcional $(\mathrm{p}<0,01)$.

Tabela 3. Pontuação no MEEM e TUG nos pacientes com DM2 e sem DM2 $(n=118)$

\begin{tabular}{lccccc}
\hline $\begin{array}{l}\text { Testes } \\
\text { funcionais }\end{array}$ & DM2 & Média \pm DP & Mediana & N & p \\
\hline MEEM (pontos) & Controle & $27,19 \pm 2,4$ & $28(19-30)$ & 68 & 0,001 \\
& DM2 & $25,14 \pm 3,4$ & $26(18-30)$ & 50 & \\
& Controle & $9,52 \pm 3,6$ & $9(6-30)$ & 56 & 0,001 \\
$\begin{array}{l}\text { Teste TUG } \\
\text { (tempo em } \\
\text { segundos) }\end{array}$ & & & & \\
& DM2 & $11,27 \pm 3,2$ & $10,5(7-20)$ & 44 & \\
\hline
\end{tabular}

Teste Mann-Whitney. DM2: diabetes melito tipo 2; MEEM: Miniexame do Estado Mental. TUG: Timed Up and Go.

Em relação ao declínio cognitivo, avaliado pelo MEEM, os pacientes DM2 apresentaram mediana de 26 pontos ( 22 a 28 ), que foi inferior à dos controles, 28 pontos (25,5 a 29), p = 0,001 (Tabela 2). No TDR, a mediana de pontos foi semelhante entre o grupo DM2 2 (1-4) e controles $3(1,5-4),(\mathrm{p}=0,121)$.

A avaliação da associação entre declínio cognitivo e dismobilidade foi positiva nos indivíduos com DM2: verificou-se pior mobilidade naqueles com maior declínio cognitivo $(\mathrm{p}=0,037)$ utilizando regressão linear. A tabela 4 demonstra que 18\% do grupo DM2 e 1,6\% do grupo controle apresentaram dismobilidade e declínio cognitivo associados. No grupo que não apresentou dismobilidade e declínio cognitivo, $57,4 \%$ eram do grupo controle $(\mathrm{p}<0,01)$.

Tabela 4. Avaliação da presença e ausência dos eventos dismobilidade e declínio cognitivo associadamente e sua correlação com DM2

\begin{tabular}{|c|c|c|c|}
\hline & Controle n (\%) & DM2 n (\%) & p \\
\hline $\begin{array}{l}\text { Presença de } \\
\text { dismobilidade e declínio } \\
\text { cognitivo }\end{array}$ & $1(1,6)$ & $11(18,0)$ & \multirow{2}{*}{$<0,01$} \\
\hline $\begin{array}{l}\text { Ausência de } \\
\text { dismobilidade e declínio } \\
\text { cognitivo }\end{array}$ & $35(57,4)$ & $14(22,9)$ & \\
\hline
\end{tabular}

\section{DISCUSSÃO}

Indivíduos com DM2 tiveram pior performance nos testes de avaliação funcional da mobilidade em relação aos controles. A população estudada, com idades entre 55 e 65 anos e predominantemente feminina, representa uma população que, mesmo independente da presença de DM2, já tem maior risco de fraturas em virtude da maior prevalência de osteoporose. Além disso, sabe-se que a população com DM2 pode apresentar maior risco de fraturas, pois as elevadas concentrações glicêmicas estão associadas ao acúmulo de produtos finais da glicação avançada (AGES) levando ao aumento da fragilidade óssea (22). Assim, a pior mobilidade funcional nesse subgrupo indica a importância desse fator na avaliação dos pacientes com DM2. Doenças osteomioarticulares têm relação com ocorrência de quedas, principalmente no tocante à redução da flexibilidade (23), mas fatores como neuropatia periférica, visão reduzida, alterações autonômicas e uso de medicamentos $(2,3,1)$, bem como sedentarismo e o ganho de peso (1), são bastante prevalentes na população com DM2. Também foi importante, no presente estudo, o maior IMC do grupo com DM2. Tal característica pode estar envolvida na pior mobilidade verificada. Em estudo realizado com uma amostra representativa da população americana (15), 73,6\% (IC 95\% 70,2-76,9) dos pacientes com DM2 relataram mais dificuldades em realizar atividades rotineiras, incluindo levantar de uma cadeira sem apoio. Neste estudo, a redução da mobilidade funcional foi correlacionada com porcentagem de hemoglobina glicada e duração da doença.

Os pacientes com DM2 também tiveram pior desempenho no TUG do que o grupo controle ( $\mathrm{p}<$ $0,05)$. A maior parte dos diabéticos $(68 \%)$ situou-se no intervalo entre 10 e 20 segundos, caracterizado como médio risco de quedas, enquanto no grupo controle a maioria $(52,9 \%)$ se enquadrou em menos de 10 segundos, com baixo risco de quedas. Em 2009, Cordeiro e cols. (20) avaliaram idosos (média de idade de 74,4 \pm 5,9 anos) com DM2 utilizando o teste TUG. O tempo médio de execução foi de $15,7 \pm 6,5$ segundos, e a maioria dos pacientes $(67,8 \%)$ levou um tempo entre 10 e 20 segundos e o restante $(21,1 \%)$ levou mais de 20 segundos, mostrando que há piora importante desse aspecto em faixas etárias maiores.

Os pacientes com DM2 apresentaram ainda pior desempenho cognitivo avaliado pelo MEEM, com mediana de 26 pontos, contra 28 pontos para os controles $(p=0,01)$. Em estudo realizado com idosos de 70 anos 
ou mais, dos quais 91\% possuíam DM2, os testes de rastreio cognitivo (entre eles, o MEEM) caracterizaram como fatores preditivos de declínio cognitivo: avanço da idade, negligência no uso de anti-hipertensivos e insulina e baixo nível de escolaridade (24). No entanto, no presente estudo a mediana de idade foi de 57 anos, e não foi verificada correlação com baixa escolaridade, possibilitando dessa forma a avaliação da presença de DM2 como principal fator determinante do declínio cognitivo nesses indivíduos. O desenho do nosso estudo avaliando apenas indivíduos que apresentavam hiperglicemia, inclusive no momento da realização dos testes cognitivos, salienta não somente a influência do DM2 na capacidade cognitiva mesmo antes dos 65 anos, como também sugere a influência da presença de hiperglicemia no desempenho do grupo com DM2.

A presença de distúrbios cognitivos e mobilidade funcional teve correlação maior nos indivíduos com DM2, e foi evidenciada pior mobilidade naqueles indivíduos com maior declínio cognitivo. Carvalho e Coutinho (12), em estudo caso-controle com pacientes de 60 anos ou mais, internados por fratura secundária a quedas, encontraram associação positiva entre demência e a ocorrência de quedas (OR: 1,82), sendo que os pacientes com demência relataram um maior número de quedas no ano anterior. Da mesma forma, o nosso estudo mostrou correlação entre distúrbios cognitivos e mobilidade funcional. No entanto, avaliamos uma população menos idosa, o que favorece a hipótese de que o DM2 influencia a mobilidade funcional e a capacidade cognitiva antes do aparecimento de complicações vasculares ou neuropáticas e sugere que a hiperglicemia é um fator agravante no desempenho de atividades que exijam funções mentais como atenção, orientação e memória de trabalho.

A pior mobilidade funcional e o pior desempenho no MEEM dos pacientes com diabetes podem advir de mau controle glicêmico, sobreposto aos demais aspectos inerentes à doença. Contudo, poucos são os estudos relacionados ao assunto em pacientes com idade inferior a 65 anos, remetendo-nos à pesquisa também em indivíduos mais jovens a fim de melhorar a qualidade de vida destes. O conhecimento sobre prejuízos na capacidade cognitiva e na mobilidade funcional dos pacientes com DM2 em faixas etárias mais jovens permite traçar estratégias de prevenção de grande importância para os programas de saúde, alertando portadores da doença e seus familiares sobre sinais de incapacidades cognitivas e físicas.
Agradecimentos: Bolsa de Iniciação Científica do Programa Institucional de Bolsas de Iniciação Científica do Conselho Nacional de Desenvolvimento Científico e Tecnológico (PIBIC/CNPq) e da Fundação Universitária do Desenvolvimento do Oeste da Fundação de Amparo à Pesquisa e Inovação do Estado de Santa Catarina (Fundeste/Fapesc).

Declaração: os autores declaram não haver conflitos de interesse científico neste estudo.

\section{REFERÊNCIAS}

1. Rejeski J, Ip EH, Bertoni AG, Bray GA, Evans G, Gregg EW, et al. Lifestyle change and mobility in obese adults with type 2 diabetes. N Engl J Med. 2012;366:1209-17.

2. Maurer MC, Burcham J, Cheng H. Diabetes mellitus is associated with an increased risk of falls in elderly residents of a long-term care facility. J Gerontology. 2005;60(9):1157-62.

3. Schwartz AV, Vittinghoff E, Sellmeyer DE, Feingold KR, Rekeneire $\mathrm{N}$, Strotmeyer ES, et al. Diabetes-related complications, glycemic control, and falls in older adults. Diabetes Care. 2008;31(3):391-6.

4. Wallace C, Reiber GE, LeMaster J, Smith DG, Sullivan K, Hayes S, et al. Incidence of falls, risk factors for falls, and fall-related fractures in individuals with diabetes and a prior foot ulcer. Diabetes Care. 2002;25(11):1983-86.

5. Schwartz AV, Hillier TA, Sellmeyer DE, Resnick HE, Gregg E, Ensrud KE, et al. Older women with diabetes have a higher risk of falls. Diabetes Care. 2002;25(10):1749-54.

6. Gregg EW, Beckles GLA, Williamson DF, Leveille SG, Langlois Ja, Engelgau MM, et al. Diabetes and physical disability among older US adults. Diabetes Care. 2000;23(9):1272-7.

7. Cox DJ, Kovachev BP, Gonder-Frederick LA, Summers KH, Clarke WL. Relationships between hyperglycemia and cognitive performance among. Diabetes Care. 2005;28(1):71-7.

8. McGuire LC, Ford ES, Ajani UA. The impact of cognitive functioning on mortality and the development of functional disability in older adults with diabetes: the second longitudinal study on aging. BMC Geriatrics. 2006;6:8.

9. Lamb SE, McCabe C, Becker C, Fried LP, Guralnik JM. The optimal sequence and selection of screening test items to predict falls risk in older disabled women: the women's health and aging study. J Gerontol A Biol Sci Med Sci. 2008;63(10):1082-8.

10. Schwartz $A V$, Villa ML, Prill M. Falls in older Mexican-American women. J Am Geriatr Soc. 1999;47(11):1371-8.

11. Podsiadlo D, Richardson S. The Time "Up \& Go": a test of basic functional mobility for frail elderly persons. J Am Ger Society. 1991;39(2):142-8.

12. DATASUS, 2008. Taxa de prevalência de diabete melito. Disponível em: http://tabnet.datasus.gov.br/cgi/dh.exe?idb2009/g01.def. Acesso em: 2 Mar, 2011.

13. Gonçalves LG, Vieira ST, Siqueira FV, Hallal PC. Prevalência de quedas em idosos asilados no município de Rio Grande, RS. Rev Saude Publica. 2008;42(5):938-45.

14. Atalaia-Silva KC, Lourenço RA. Tradução, adaptação e validação de construto do teste do relógio aplicado entre idosos no Brasil. Rev Saude Publica. 2008;42(5)930-7.

15. Kalyani RR, Saudek CD, Brancati FL, Selvin E. Association of diabetes, comorbidities, and $\mathrm{A} 1 \mathrm{c}$ with functional disability in older adults. Diabetes Care. 2010;33(5):1055-60.

16. Salarian A, Horak FB, Zampieri C, Kuhta P, Nutt JG, Aminian K. ITUG, a sensitive and reliable measure of mobility. IEEE Trans Neural Syst Rehabil Eng. 2010;18(3):303-10. 
17. Guimarães LHCT, Galdino DCA, Martins FLM, Vitorino DFM, Pereira KL, Carvalho EM. Comparação da propensão de quedas entre idosos que praticam atividade física e idosos sedentários. Rev Neurociencias. 2004;12(2):68-72.

18. Bohannon RW. Reference values for the time up and go test: a descriptive meta-analysis. J Geriatric Physical Therapy. 2006;29(2) 64-8.

19. Alvarenga KF, Duarte JL, Silva DPC, Agostinho-Pesse RS, Negrato CA, Costa AO. Potencial cognitivo P300 em indivíduos com diabetes mellitus. Rev Bras Otorrinolaringol. 2005;71(2):202-5.

20. Cordeiro RC, Jardim JR, Perracini MR, Ramos LR. Factors associated with functional balance and mobility among elderly diabetic outpatients. Arq Bras Endocrinol Metab. 2009;53(7):83443.
21. Lourenço RA, Veras RP. Mini-Exame do Estado Mental: características psicométricas em idosos ambulatoriais. Rev Saude Publica. 2006;40(4):712-9.

22. Shane E, Burr D, Ebeling PR, Abrahamsen B, Adler RA, Brown TD, et al. Atypical subtrochanteric and diaphyseal femoral fractures: report of a task force of the American Society for Bone and Mineral Research. J Bone Mineral Research. 2010;25(11):2267-94.

23. Guimarães JMN, Farinatti PTV. Análise descritiva de variáveis teoricamente associadas ao risco de quedas em mulheres idosas. Rev Bras Med Esporte. 2005;11(5)299-305.

24. Bruce DG, Davis WA, Casey GP, Starkstein SE, Clarnette RM, Almeida OP, et al. Predictors of cognitive decline in older individuals with diabetes. Diabetes Care. 2008;31(11):460-72. 